\title{
Zoll metrics, branched covers, and holomorphic disks
}

\author{
Claude LeBrun and L.J. Mason
}

\begin{abstract}
We strengthen our previous results [12] regarding the moduli spaces of Zoll metrics and Zoll projective structures on $S^{2}$. In particular, we describe a concrete, open condition which suffices to guarantee that a totally real embedding $\mathbb{R P}^{2} \hookrightarrow \mathbb{C P}_{2}$ arises from a unique Zoll projective structure on the two-sphere. Our methods ultimately reflect the special role such structures play in the initial value problem for the three-dimensional Lorentzian Einstein-Weyl equations.
\end{abstract}

A Zoll metric on a smooth manifold $M$ is a Riemannian metric $g$ whose geodesics are all simple closed curves of equal length. This terminology commemorates the fundamental contribution of Otto Zoll [21], who exhibited an infinite-dimensional family of such metrics on $M=S^{2}$. It is easy to prove [2] that a manifold admitting Zoll metrics is compact and has finite fundamental group, so the only two-dimensional candidates for $M$ are $S^{2}$ and $\mathbb{R P}^{2}$; conversely, the standard metrics on both of these surfaces are obviously Zoll. However, Green's proof [7] of the Blaschke conjecture shows that, after rescaling, every Zoll metric on $\mathbb{R P}^{2}$ is actually a pull-back of the standard one via some diffeomorphism. By contrast, Zoll's examples show that the situation for the two-sphere is fundamentally different. Indeed, in the decade following Zoll's work, Funk [6] gave a formal-power-series argument indicating that, modulo isometries and rescalings, the general Zoll perturbation of the standard metric on $S^{2}$ depends on one odd function $f: S^{2} \rightarrow \mathbb{R}$. However, a rigorous proof of Funk's conjectural picture was only found half a century later, when Victor Guillemin [10] brought the power of Nash-Moser implicit function theorems to bear on the problem.

More recently, twistor techniques have given us new insights into global aspects of the problem. Indeed, the present authors have elsewhere shown [12] that Zoll surfaces can, in principle, be completely understood in terms of families of holomorphic disks in $\mathbb{C P}_{2}$. These same techniques are also naturally adapted to the study of more general Zoll projective structures. Recall that a projective structure is by definition an equivalence class [ $\nabla]$ of 
affine connections $\nabla$ on a manifold $M$, where two connections are declared to be equivalent iff they have the same geodesics, considered as unparameterized curves. A projective structure is said to be Zoll iff its geodesics (again, as unparameterized curves) are all embedded circles. It can then be shown $[8,12]$ that a Riemannian metric $g$ on a compact surface $M$ is Zoll iff the equivalence class $[\nabla]$ of its Levi-Civita connection is a Zoll projective structure. A compact surface $M$ can admit a Zoll projective structure $[\nabla]$ iff it is diffeomorphic to $S^{2}$ or $\mathbb{R P}^{2}$; and, as in the Riemannian case, any Zoll projective structure on $\mathbb{R P}^{2}$ is actually the standard one, pulled back via some self-diffeomorphism of $\mathbb{R P}^{2}$. Our proof of this last assertion [12] hinged on the fact that the complex structure of $\mathbb{C P}_{2}$ is unique [19] up to biholomorphism.

We now summarize our previous results [12] regarding the case of $M=$ $S^{2}$. Given a smooth Zoll projective structure $[\nabla]$ on $M$, its space of unoriented geodesics $N \approx \mathbb{R P}^{2}$ has a natural embedding in $\mathbb{C P}_{2}$ as a totally real submanifold, in a manner which is completely determined up to a projective linear transformation; for example, the usual projective structure induced by the standard "round" metric corresponds to a "real linear" embedding $\mathbb{R P}^{2} \hookrightarrow \mathbb{C P}_{2}$. Each point $x \in M$ determines an embedded holomorphic disk $\Delta_{x} \subset \mathbb{C P}_{2}$ with $\partial \Delta_{x} \subset N$, and the relative homology class $\left[\Delta_{x}\right]$ of any such disk generates $H_{2}\left(\mathbb{C P}_{2}, N ; \mathbb{Z}\right) \approx \mathbb{Z}$. These disks meet $N$ only along their boundaries, and their interiors foliate $\mathbb{C P}_{2}-N$. The family of disks $\Delta_{x}$ moreover sweeps out an entire connected component in the moduli space of holomorphic disks $\left(D^{2}, \partial D^{2}\right) \rightarrow\left(\mathbb{C P}_{2}, N\right)$. If the family of disks $\left\{\Delta_{x} \mid x \in M\right\}$ is known, the projective structure $[\nabla]$ can then be completely reconstructed; namely, given a point $z \in N$, the set

$$
\mathfrak{C}_{z}=\left\{x \in M \mid z \in \partial \Delta_{x}\right\}
$$

is a geodesic of $[\nabla]$, and every geodesic arises in this way.

The construction proceeds by first creating an abstract complex surface, and then showing that it must be biholomorphic to $\mathbb{C P}_{2}$. In the process, the bundle of orientation-compatible almost-complex structures over $M=$ $S^{2}$ is identified with the complement $\mathbb{C P}_{2}-N$ of the relevant totally real submanifold $N$. If there is an orientation-compatible complex structure $J$ on $M$ which is parallel with respect to some torsion-free connection $\nabla \in$ $[\nabla]$, then the graph of $J$ becomes a holomorphic curve $\mathcal{Q} \subset \mathbb{C P}_{2}-N$. For homological reasons, this curve must be a non-singular conic, and so may be put in the standard form

$$
z_{1}^{2}+z_{2}^{2}+z_{3}^{2}=0
$$


by making a suitable choice of homogeneous coordinates on $\mathbb{C P}_{2}$. Notice that this happens precisely when there is a conformal structure $[g]$ on $M$ for which $\nabla$ is a compatible Weyl connection. If there is actually a Zoll metric $g$ with Levi-Civita connection $\nabla \in[\nabla]$, then the totally real submanifold $N \subset \mathbb{C P}_{2}$ is moreover Lagrangian with respect to the sign-ambiguous symplectic form $\Omega=\Im m \Upsilon$ on $\mathbb{C P}_{2}-\mathcal{Q}$, where

$$
\Upsilon= \pm \frac{z_{1} d z_{2} \wedge d z_{3}+z_{2} d z_{3} \wedge d z_{1}+z_{3} d z_{1} \wedge d z_{2}}{\sqrt{\left(z_{1}^{2}+z_{2}^{2}+z_{3}^{2}\right)^{3}}}
$$

In the converse direction, one would like to assert that the totally real submanifold $N \subset \mathbb{C P}_{2}$ can be chosen essentially arbitrarily, and that each such choice uniquely determines a Zoll projective structure [ $\nabla]$ on $M=S^{2}$. But while our previous results in this direction may have been conceptually suggestive, they were technically crude in important respects. Indeed, using an elementary inverse-function theorem argument, we merely showed in [12] that every $N \subset \mathbb{C P}_{2}$ which is close to the standard "real linear" $\mathbb{R P}^{2}$ in the $C^{2 k+5}$ topology actually arises from a $C^{k}$ Zoll projective structure $[\nabla]$, and that this projective structure is unique among those that are close to the standard "round" projective structure. By contrast, the rest of the story was quite clean; the choice of a reference conic $\mathcal{Q} \subset \mathbb{C P}_{2}$ disjoint from such an $N$ then gives rise to a conformal structure $[g]$ on $M=S^{2}$ for which the Zoll projective structure $[\nabla]$ is represented by a unique $[g]$-compatible Weyl connection $\nabla \in[\nabla]$, and this Weyl connection is the Levi-Civita connection of a Zoll metric $g \in[g]$ iff $N$ is Lagrangian with respect to the signambigious symplectic form $\Omega$. Still, it must be admitted that our previous results remain esthetically unsatisfactory in two essential ways: we neither provided an effective condition on $N \subset \mathbb{C P}_{2}$ sufficient for the existence of an associated family of holomorphic disks, nor proved the uniqueness of this family when it does exist.

The present article will address these issues by proving global existence and uniqueness results for holomorphic disks; see Theorems 1 to 3 below. For the sake of clarity, our discussion is set almost entirely in the smooth $\left(C^{\infty}\right)$ context. While our present methods certainly afford us this luxury, the interested reader may nonetheless wish to verify that most of our arguments can in fact be carried out with much less regularity. The reader may also find it interesting to compare and contrast our uniqueness results with the rather different ones found in [17].

We now begin by fixing the standard non-singular conic $\mathcal{Q} \subset \mathbb{C P}_{2}$ given by (1). Of course, any two non-singular conics are actually projectively 
equivalent, but our conventional choice of $\mathcal{Q}$ has the nice additional feature that it is manifestly invariant under an anti-holomorphic involution

$$
\begin{aligned}
\mathfrak{c}: \mathbb{C P}_{2} & \longrightarrow \mathbb{C P}_{2}, \\
{\left[z_{1}, z_{2}, z_{3}\right] } & \longmapsto\left[\bar{z}_{1}: \bar{z}_{2}: \bar{z}_{3}\right],
\end{aligned}
$$

whose fixed-point set is disjoint from $\mathcal{Q}$. This fixed-point set will henceforth be called the standard $\mathbb{R P}^{2} \subset \mathbb{C P}_{2}$.

Next, notice that a projective line $\mathcal{A} \subset \mathbb{C P}_{2}$ is tangent to $\mathcal{Q}$ iff it is given by

$$
a_{1} z_{1}+a_{2} z_{2}+a_{3} z_{3}=0
$$

for an element $\left[a_{1}: a_{2}: a_{3}\right]$ of the dual projective plane $\mathbb{C P}_{2}^{*}$ satisfying

$$
a_{1}^{2}+a_{2}^{2}+a_{3}^{2}=0 .
$$

When this happens, the point of tangency is then given by

$$
\left[z_{1}: z_{2}: z_{3}\right]=\left[a_{1}: a_{2}: a_{3}\right] .
$$

Also notice that if $p=\left[p_{1}: p_{2}: p_{3}\right]$ belongs to the complement of $\mathcal{Q}$ in $\mathbb{C P}_{2}$, there are always exactly two tangent lines of $\mathcal{Q}$ which pass through $p$; indeed, the incidence equation

$$
a_{1} p_{1}+a_{2} p_{2}+a_{3} p_{3}=0
$$

for $\left[a_{1}: a_{2}: a_{3}\right]$ describes a line in the dual projective plane $\mathbb{C P}_{2}^{*}$ which is not tangent to the dual conic (4), and which therefore, by Bézout's theorem, must meet the dual conic in two distinct points.

The standard $\mathbb{R P}^{2} \subset \mathbb{C P}_{2}$ is an example of a totally real submanifold. If $Z$ is a complex manifold with integrable almost-complex structure $J$, recall that a differentiable submanifold $S \subset Z$ is said to be (maximally) totally real if $\left.T Z\right|_{S}=T S \oplus J(T S)$. We now introduce a special class of totally real surfaces in $\mathbb{C P}_{2}$ that will be central to our discussion.

Definition 1. A compact connected smoothly embedded two-manifold $N \subset \mathbb{C P}_{2}$ will be called a docile surface if

- $N$ is a totally real submanifold of $\mathbb{C P}_{2}$;

- $N$ is disjoint from the conic $\mathcal{Q}$ defined by (1); and

- $N$ is transverse to each tangent projective line (4) of the conic $\mathcal{Q}$. 
For example, the standard $\mathbb{R P}^{2} \subset \mathbb{C P}_{2}$ is docile. Indeed, the transversality condition is satisfied in this case because a projective line is tangent to this standard $\mathbb{R P}^{2}$ iff it is invariant under complex conjugation, whereas the two tangent lines of $\mathcal{Q}$ which pass through a real point $p \in \mathbb{R P}^{2}$ are, by contrast, interchanged by the anti-holomorphic involution $\mathfrak{c}$ defined above in $(3)$.

Since the condition of docility is obviously open in the $C^{1}$ topology, any small perturbation of the standard $\mathbb{R P}^{2}$ will also be a docile surface. In the converse direction, we have the following result:

Lemma 1. Let $N \subset \mathbb{C P}_{2}$ be a docile surface. Then $N$ is diffeomorphic to $\mathbb{R P}^{2}$, and is isotopic to the standard $\mathbb{R P}^{2} \subset \mathbb{C P}_{2}$ through a family of other docile surfaces.

Proof. The argument will proceed by systematically exploiting the map

$$
\begin{aligned}
\Pi: \mathbb{C P}_{1} \times \mathbb{C P}_{1} & \rightarrow \mathbb{C P}_{2} \\
\left(\left[u_{1}: u_{2}\right],\left[v_{1}: v_{2}\right]\right) & \mapsto\left[i\left(u_{1} v_{1}+u_{2} v_{2}\right): u_{1} v_{1}-u_{2} v_{2}: u_{1} v_{2}+u_{2} v_{1}\right]
\end{aligned}
$$

which is a two-to-one branched cover, ramified over the conic $\mathcal{Q}$. Indeed, notice that the diagonal $\mathcal{D}$, explicitly given by $u_{1} v_{2}-v_{1} u_{2}=0$, is sent bijectively to the conic $z_{1}^{2}+z_{2}^{2}+z_{3}^{2}=0$. Moreover, each factor line $u=$ const or $v=$ const is sent isomorphically to a tangent line $\mathcal{A}$ of $\mathcal{Q}$, and every tangent line conversely occurs in each of these families. Since $\Pi$ has degree two, and since exactly two tangent lines pass through each $p \in \mathbb{C P}_{2}-\mathcal{Q}$, it follows that $\Pi$ is actually unramified away from $\mathcal{Q}$. We note in passing that the "anti-diagonal" $\overline{\mathcal{D}}$, defined by $\left[u_{1}: u_{2}\right]=\left[-\bar{v}_{2}: \bar{v}_{1}\right]$, is therefore a two-to-one cover of the standard $\mathbb{R P}^{2} \subset \mathbb{C P}_{2}$ defined by $z_{j}=\bar{z}_{j}, j=1,2,3$.

Now suppose that $N \subset \mathbb{C P}_{2}$ is a docile surface, and let $\tilde{N}=\Pi^{-1}(N)$ be its pre-image in $\mathbb{C P}_{1} \times \mathbb{C P}_{1}$. Since $N \cap \mathcal{Q}=\varnothing$, it follows that $\tilde{N}$ is a smooth compact surface in $\mathbb{C P}_{1} \times \mathbb{C P}_{1}$, and the induced map $\tilde{N} \rightarrow N$ is a two-to-one submersion. In particular, $\tilde{N}$ has at most two connected components. On the other hand, the transversality hypothesis guarantees that $\tilde{N}$ is transverse to each factor line $u=$ const or $v=$ const, so each factor projection

$$
\left.\varpi_{j}\right|_{\tilde{N}}: \tilde{N} \rightarrow \mathbb{C P}_{1}, \quad j=1,2
$$

is a submersion, and hence a covering map on each connected component. Since $\mathbb{C P}_{1}$ is simply connected, both projections are therefore diffeomorphisms on each connected component, and each component of $\tilde{N}$ is therefore 
the graph of some diffeomorphism $\varphi: \mathbb{C P}_{1} \rightarrow \mathbb{C P}_{1}$. Moreover, since $\tilde{N} \cap \mathcal{D}=$ $\varnothing$, the relevant $\varphi$ cannot have fixed points, and is therefore homotopic to the antipodal map

$$
\begin{aligned}
& \mathfrak{a}: \mathbb{C P}_{1} \longrightarrow \mathbb{C P}_{1}, \\
& {\left[z_{1}: z_{2}\right] \longmapsto\left[-\bar{z}_{2}: \bar{z}_{1}\right]}
\end{aligned}
$$

via the familiar geometric construction of pushing $\varphi(z)$ away from $z$ along great circles. Consequently, each such diffeomorphism $\varphi$ has degree -1 , and so is orientation-reversing.

Thus, if $\tilde{N}$ were disconnected, it would have to be the union of two disjoint graphs of orientation-reversing diffeomorphisms $\varphi_{1}, \varphi_{2}: \mathbb{C P}_{1} \rightarrow \mathbb{C P}_{1}$. Since these two graphs would be disjoint, we would have $\varphi_{1}(u) \neq \varphi_{2}(u)$, and hence $\left(\varphi_{2}^{-1} \circ \varphi_{1}\right)(u) \neq u$, for all $u \in \mathbb{C P}_{1}$. Thus $\varphi_{2}^{-1} \circ \varphi_{1}$ would be fixedpoint-free, and hence would also have degree -1 . But since

$$
\operatorname{deg}\left(\varphi_{2}^{-1} \circ \varphi_{1}\right)=\left(\operatorname{deg} \varphi_{2}\right)^{-1}\left(\operatorname{deg} \varphi_{1}\right)=(-1)^{2}=+1,
$$

this is clearly a contradiction.

Hence $\tilde{N}$ is connected, and therefore the graph of a single fixed-pointfree, orientation-reversing diffeomorphism $\varphi: \mathbb{C P}_{1} \rightarrow \mathbb{C P}_{1}$. However, by construction, $\tilde{N}=\Pi^{-1}(N)$ is invariant under the holomorphic involution

$$
\begin{aligned}
\varrho: \mathbb{C P}_{1} \times \mathbb{C P}_{1} & \longrightarrow \mathbb{C P}_{1} \times \mathbb{C P}_{1}, \\
(u, v) & \longmapsto(v, u)
\end{aligned}
$$

and every $(u, \varphi(u))$ must therefore also be expressible as $(\varphi(v), v)$. Thus $\varphi=\varphi^{-1}$, and $\varphi^{2}=$ id. By projection to the first factor, the action of the non-trivial deck transformation on $\tilde{N}$ can therefore be identified with the action of $\varphi$ on $\mathbb{C P}_{1}$, and the quotient $N$ of $\tilde{N}$ by this deck transformation can therefore be identified with $\mathbb{C P}_{1} /\langle\varphi\rangle$. But this is a smooth compact surface with fundamental group $\mathbb{Z}_{2}$, and so necessarily diffeomorphic to $\mathbb{R P}^{2}=\mathbb{C P}_{1} /\langle\mathfrak{a}\rangle$. Lifting some diffeomorphism $\psi_{0}: \mathbb{C P}_{1} /\langle\mathfrak{a}\rangle \rightarrow \mathbb{C P}_{1} /\langle\varphi\rangle$ to an orientation-preserving diffeomorphism $\psi: \mathbb{C P}_{1} \rightarrow \mathbb{C P}_{1}$ of universal covers now shows that we must have

$$
\varphi=\psi \circ \mathfrak{a} \circ \psi^{-1}
$$

for some orientation-preserving diffeomorphism $\psi$ of $\mathbb{C P}_{1}$.

This conclusion can now be reverse-engineered. Indeed, given any orientation-preserving diffeomorphism $\psi: \mathbb{C P}_{1} \rightarrow \mathbb{C P}_{1}$, the graph of the 
orientation-reversing involution $\varphi=\psi \circ \mathfrak{a} \circ \psi^{-1}$ necessarily projects, via $\Pi$, to a docile surface in $\mathbb{C P}_{2}$. However, the group of orientation-preserving diffeomorphisms of $\mathbb{C P}_{1}$ is connected, since it acts transitively on the (connected) space of conformal metrics on $S^{2}$, with (connected) isotropy subgroup $P S L(2, \mathbb{C})$. It therefore follows that the space of docile surfaces in $\mathbb{C P}_{2}$ is also connected. In particular, any given docile surface $N$ may be smoothly deformed, via a family of docile surfaces, into the standard $\mathbb{R P}^{2} \subset \mathbb{C P}_{2}$.

Lemma 2. Let $N \subset \mathbb{C P}_{2}$ be any docile surface. Then the homomorphism $H_{2}\left(\mathbb{C P}_{2}, N\right) \rightarrow \mathbb{Z}$ given by homological intersection with $[\mathcal{Q}] \in H_{2}\left(\mathbb{C P}_{2}-N\right)$ is an isomorphism. In particular, $\mathrm{H}_{2}\left(\mathbb{C P}_{2}, N\right) \cong \mathbb{Z}$.

Proof. Let us first observe that there is a homeomorphism $\Psi: \mathbb{C P}_{2} \rightarrow \mathbb{C P}_{2}$ which sends $\mathbb{R P}_{2}$ to $N$, and $\mathcal{Q}$ to itself. Indeed, let $\psi: \mathbb{C P}_{1} \rightarrow \mathbb{C P}_{1}$ be a diffeomorphism such that $\tilde{N}$ is the graph of $\psi \circ \mathfrak{a} \circ \psi^{-1}$, and then define $\tilde{\Psi}:\left(\mathbb{C P}_{1} \times \mathbb{C P}_{1}\right) \rightarrow\left(\mathbb{C P}_{1} \times \mathbb{C P}_{1}\right)$ to be $\psi \times \psi$. Then $\tilde{\Psi}$ send $\overline{\mathcal{D}}$ to $\tilde{N}$, and $\mathcal{D}$ to itself, while commuting with the involution $\varrho$ given by $\varrho(u, v)=(v, u)$. Since $\varrho$ acts transitively on the fibers of $\Pi$, and since $\Pi: \mathbb{C P}_{1} \times \mathbb{C P}_{1} \rightarrow \mathbb{C P}_{2}$ is a quotient map, there is a unique homeomorphism $\Psi: \mathbb{C P}_{2} \rightarrow \mathbb{C P}_{2}$ such that $\Pi \circ \tilde{\Psi}=\Psi \circ \Pi$. This homeomorphism $\Psi$ then sends $\mathbb{R P}_{2}$ to $N$, and $\mathcal{Q}$ to $\mathcal{Q}$, as promised, and preserves the orientations of both $\mathbb{C P}_{2}$ and $\mathcal{Q}$.

Thus $H_{2}\left(\mathbb{C P}_{2}, N\right) \cong H_{2}\left(\mathbb{C P}_{2}, \mathbb{R P}^{2}\right)$, and we only really need to check the claim when $N=\mathbb{R P}^{2}$. However, $\mathbb{C P}_{2}$ is the union of a two-disk bundle $X \rightarrow \mathbb{R P}^{2}$ and a two-disk bundle $Y \rightarrow \mathcal{Q}$, identified along their boundaries. (This can be checked by hand [12], but it can more elegantly be deduced [9] from the fact that there is a cohomogeneity-one action of $S O(3)$ on $\mathbb{C P}_{2}$, with exceptional orbits $\mathbb{R P}^{2}$ and $\mathcal{Q}$.) Thus $H_{2}\left(\mathbb{C P}_{2}, \mathbb{R P}^{2}\right) \cong H_{2}\left(\mathbb{C P}_{2}, X\right)$ by homotopy equivalence, whereas $H_{2}\left(\mathbb{C P}_{2}, X\right) \cong H_{2}(Y, \partial Y)$ by excision, and $H_{2}(Y, \partial Y) \cong H^{2}(Y)$ by Lefschetz-Poincaré duality. Since $\mathcal{Q}$ is a deformation retract of $Y$, it follows that $H_{2}\left(\mathbb{C P}_{2}, N\right) \cong H^{2}(\mathcal{Q})=\mathbb{Z}$. Moreover, since $[\mathcal{Q}]$ generates $H_{2}(Y) \cong \mathbb{Z}$, Lefschetz-Poincaré duality guarantees that homological intersection with $[\mathcal{Q}]$ is an isomorphism $H_{2}\left(\mathbb{C P}_{2}, N\right) \rightarrow \mathbb{Z}$.

Remark 1. The homeomorphism $\Psi$ constructed above will typically fail to be smooth along $\mathcal{Q}$. However, it is not difficult to modify $\Psi$ to produce a self-diffeomorphism of $\mathbb{C P}_{2}$ with all the properties in question. We leave this exercise as a challenge to the interested reader.

Lemma 3. Let $N \subset \mathbb{C P}_{2}$ be any docile surface, and let $\tilde{N} \subset \mathbb{C P}_{1} \times \mathbb{C P}_{1}$ be its inverse image under $\Pi$. Then $\mathbb{C P}_{1} \times \mathbb{C P}_{1}$ admits a $\varrho$-invariant Kähler 
metric $h$ for which $\tilde{N}$ is Lagrangian. This metric can be chosen so that its Kähler form $\omega$ represents $2 \pi c_{1}\left(\mathbb{C P}_{1} \times \mathbb{C P}_{1}\right)$ in deRham cohomology, and if $N$ is smoothly varied through a family of other docile surfaces, a corresponding family of such Kähler metrics can moreover be chosen so as to depend smoothly on the given parameters.

Proof. The construction is a variant of one used in [13, Lemma 2]. Express $\tilde{N}$ as the graph of a smooth orientation-reversing involution $\varphi: \mathbb{C P}_{1} \rightarrow \mathbb{C P}_{1}$. Let $\alpha$ be the area form of the standard unit-sphere metric on $S^{2}=\mathbb{C P}_{1}$. Then the area form

$$
\check{\omega}=\frac{\alpha-\varphi^{*} \alpha}{2}
$$

satisfies $\varphi^{*} \check{\omega}=-\check{\omega}$, and is the Kähler form of a unique Kähler metric $\check{h}$ on $\mathbb{C P}_{1}$. We now define a Kähler metric on $\mathbb{C P}_{1} \times \mathbb{C P}_{1}$ by setting

$$
h=\varpi_{1}^{*} \check{h}+\varpi_{2}^{*} \check{h},
$$

where $\varpi_{j}: \mathbb{C P}_{1} \times \mathbb{C P}_{1} \rightarrow \mathbb{C P}_{1}, j=1,2$, are the factor projections. Since the restriction of the associated Kähler form $\omega$ to the graph of $\varphi$ is

$$
(\operatorname{id} \times \varphi)^{*}\left(\varpi_{1}^{*} \check{\omega}+\varpi_{2}^{*} \check{\omega}\right)=\check{\omega}+\varphi^{*} \check{\omega}=\check{\omega}-\check{\omega}=0,
$$

the graph $\tilde{N}$ is Lagrangian with respect to $\omega$. Moreover, $h$ is invariant under the interchange of the factors of $\mathbb{C P}_{1} \times \mathbb{C P}_{1}$, and the Kähler class of $h$ is obviously given by $[\omega]=2 \pi c_{1}\left(\mathbb{C P}_{1} \times \mathbb{C P}_{1}\right)$, since $\omega$ has integral $4 \pi$ on either factor $\mathbb{C P}_{1}$. Finally, this construction can be uniformly applied to a smooth family $N_{t}$ of docile surfaces by replacing $\varphi$ with a corresponding of smooth family $\varphi_{t}$ of smooth involutions, and the corresponding family $h_{t}$ of Kähler metrics will then manifestly depend smoothly on the parameter $t$.

Definition 2. Let $D^{2}$ denote the closed unit disk in $\mathbb{C}$, and let $Z$ be any complex manifold. A continuous map $f: D^{2} \rightarrow Z$ will be called a parameterized holomorphic disk in $Z$ if $f$ is holomorphic in the open unit disk $\stackrel{\circ}{D}^{2}=D^{2}-\partial D^{2}$. If, in addition, $f(\partial D) \subset W$ for a specified subset $W \subset Z$, we will sometimes say that $f$ is a parameterized holomorphic disk in $(Z, W)$.

Proposition 1. Let $N \subset \mathbb{C P}_{2}$ be any docile surface, and suppose that $f$ is a parameterized holomorphic disk in $\left(\mathbb{C P}_{2}, N\right)$ whose relative homology class $[f]$ generates $H_{2}\left(\mathbb{C P}_{2}, N\right) \cong \mathbb{Z}$. Then $f$ is a smooth embedding, $f\left(D^{2}\right)$ meets $N$ only along $f\left(\partial D^{2}\right)$, and $f\left(D^{2}\right)$ meets $\mathcal{Q}$ transversely, in a single point. 
Proof. Because $\left.f\right|_{\partial D^{2}}$ takes values in the totally real submanifold $N$, and the latter is assumed to be a submanifold of class $C^{\infty}$, the holomorphic map $f$ must actually be smooth up to the boundary $[1,3]$. Since we have also assumed that $[f]$ generates $H_{2}\left(\mathbb{C P}_{2}, N\right) \cong \mathbb{Z}$, its homological intersection number with $\mathcal{Q}$ must be 1 by Lemma 2, and the disk $f$ can therefore only geometrically intersect $\mathcal{Q}$ transversely, in a single point (which we may assume to be $f(0)$ ), since every geometric intersection of distinct holomorphic curves makes a contribution with positive multiplicity toward their total homological intersection number.

Now, because $\Pi: \mathbb{C P}_{1} \times \mathbb{C P}_{1} \rightarrow \mathbb{C P}_{2}$ is a two-to-one branched cover, ramified only at $\mathcal{Q}$, path-lifting of the null-homotopic circles $f\left(r \mathrm{e}^{2 \mathrm{i} \theta}\right)$ in $\mathbb{C P}_{2}-\mathcal{Q}$ allows us to construct a continuous lift $\tilde{f}: D^{2} \rightarrow \mathbb{C P}_{1} \times \mathbb{C P}_{1}$ with $\Pi(\tilde{f}(\zeta))=f\left(\zeta^{2}\right)$. Since this lift is moreover holomorphic away from the origin, it then follows that $\tilde{f}$ is also holomorphic across the origin by the Riemann removable singularities theorem. We thus obtain a parameterized holomorphic disk $\tilde{f}: D^{2} \rightarrow \mathbb{C P}_{1} \times \mathbb{C P}_{1}$ which sends $\partial D^{2}$ to $\tilde{N}=\Pi^{-1}(N)$ and 0 to the unique $\tilde{p} \in \mathcal{D}$ with $\Pi(\tilde{p})=p$, while satisfying $\tilde{f}(-\zeta)=\varrho(\tilde{f}(\zeta))$, where $\varrho$ is once again the involution $(u, v) \mapsto(v, u)$ of $\mathbb{C P}_{1} \times \mathbb{C P}_{1}$. Also note that, given any $\tilde{f}$ satisfying these properties, one may conversely construct a disk $f$ in $\mathbb{C P}_{2}$ with the desired properties by setting $f(\zeta)=\Pi(\tilde{f}( \pm \sqrt{\zeta}))$, since this well-defined map is obviously continuous, and is holomorphic away from the origin.

Now, given a holomorphic disk $f$ representing the generator of $H_{2}\left(\mathbb{C P}_{2}, N\right)$, the associated disk $\tilde{f}$ will then represent the generator of $H_{2}\left(\mathbb{C P}_{1} \times \mathbb{C P}_{1}, \tilde{N}\right) \cong \mathbb{Z}$. Indeed, since $\tilde{N}$ is homotopic to the anti-diagonal $\overline{\mathcal{D}}$ in $\left(\mathbb{C P}_{1} \times \mathbb{C P}_{1}\right)-\mathcal{D}$, the long exact sequence

$$
\cdots \rightarrow H_{2}(\tilde{N}) \rightarrow H_{2}\left(\mathbb{C P}_{1} \times \mathbb{C P}_{1}\right) \rightarrow H_{2}\left(\mathbb{C P}_{1} \times \mathbb{C P}_{1}, \tilde{N}\right) \rightarrow H_{1}(\tilde{N}) \rightarrow \cdots
$$

implies that the diagonal class $[\mathcal{D}]$ will necessarily represent twice the generator of $H_{2}\left(\mathbb{C P}_{1} \times \mathbb{C P}_{1}, \tilde{N}\right)$. However, $\Pi(\mathcal{D})$ is the degree-2 holomorphic curve $\mathcal{Q}$, so $\Pi_{*}: H_{2}\left(\mathbb{C P}_{1} \times \mathbb{C P}_{1}, \tilde{N}\right) \rightarrow H_{2}\left(\mathbb{C P}_{2}, N\right)$ is therefore the homomorphism $\mathbb{Z} \rightarrow \mathbb{Z}$ given by multiplication by 2 . But $\Pi_{*}([\tilde{f}])=2[f]$ by construction, so it follows that $[\tilde{f}]$ is indeed the generator of $H_{2}\left(\mathbb{C P}_{1} \times \mathbb{C P}_{1}, \tilde{N}\right)$, as claimed. In particular, since $\tilde{N}$ is Lagrangian with respect the Kähler form $\omega$ of the metric $h$ constructed in Lemma 3, we must have

$$
\int_{D^{2}} \tilde{f}^{*} \omega=\frac{1}{2} \int_{\mathcal{D}} \omega=\frac{1}{2} \int_{\mathcal{D}} 2 \pi c_{1}=\frac{1}{2} 2 \pi(4)=4 \pi .
$$

Next, by making precise an argument previously sketched in [13, Lemma 3 ], we will show that $\tilde{f}$ must be a topological embedding. Indeed, consider 
the abstract oriented two-sphere obtained by taking the double $D^{2} \cup \overline{D^{2}}$, where the two copies of the disk are identified along the boundary, $D^{2}$ is given the usual orientation coming from the unit disk in $\mathbb{C}$ and $\overline{D^{2}}$ is given the opposite orientation. Given $f$ as above, we can then construct a continuous map $F: D^{2} \cup \overline{D^{2}} \rightarrow \mathbb{C P}_{1}$, defined to equal $\varpi_{1} \circ \tilde{f}$ on $D^{2}$, and to equal $\varphi^{-1} \circ \varpi_{2} \circ \tilde{f}$ on $\overline{D^{2}}$. Note that $F$ is actually smooth when restricted to either $D^{2}$ or $\overline{D^{2}}$, and that it is orientation preserving at every regular point of the interior of either two-disk hemisphere. The complex dilatation $\mu$ of $F$ is bounded by that of $\varphi^{-1}$, so $F$ is consequently quasi-regular in the sense of [16]. However, via the measurable Riemann mapping theorem, this implies [14, Section VI.2.3] that $F=G \circ H$ for some quasi-conformal homeomorphism $H: D^{2} \cup \overline{D^{2}} \rightarrow \mathbb{C P}_{1}$ and some holomophic map $G: \mathbb{C P}_{1} \rightarrow \mathbb{C P}_{1}$.

However, equipping $\mathbb{C P}_{1}$ and $\mathbb{C P}_{1} \times \mathbb{C P}_{1}$, respectively, with the Kähler forms $\check{\omega}$ and $\omega$ used in the proof of Lemma 3 , we have

$$
\int_{D^{2} \cup \overline{D^{2}}} F^{*} \check{\omega}=\int_{D^{2}} \tilde{f}^{*}\left(\varpi_{1}^{*} \check{\omega}+\varpi_{2}^{*} \check{\omega}\right)=\int_{D^{2}} \tilde{f}^{*} \omega=4 \pi=\int_{\mathbb{C P}_{1}} \check{\omega}
$$

and it therefore follows that the piece-wise smooth map $F$ has degree 1 . The holomorphic map $G: \mathbb{C P}_{1} \rightarrow \mathbb{C P}_{1}$ is therefore a biholomorphism, and $F$ is therefore a quasi-conformal homeomorphism. In particular, $\varpi_{j} \circ \tilde{f}$ must be a homeomorphism for $j=1,2$, and $\left(\varpi_{1} \circ \tilde{f}\right)\left(\stackrel{\circ}{D}^{2}\right)$ must be disjoint from $\left(\varphi^{-1} \circ \varpi_{2} \circ \tilde{f}\right)\left(\stackrel{\circ}{D}^{2}\right)$. In particular, the graph $\tilde{N}$ of $\varphi$ is disjoint from $\tilde{f}\left(\stackrel{\circ}{ }^{2}\right)$, and hence $N$ is disjoint from $f\left(\stackrel{\circ}{D}^{2}\right)$, too.

To finish the proof, we will now show that the smooth maps

$$
\varpi_{j} \circ \tilde{f}: D^{2} \rightarrow \mathbb{C P}_{1}, j=1,2
$$

are actually smooth embeddings. Of course, since we already know that they are homeomorphisms, and since they are manifestly holomorphic on the interior $\stackrel{\circ}{D}^{2}$ of the disk, it only remains to show that these maps are immersions along $\partial D^{2}$. However, since $\tilde{N} \subset \mathbb{C P}_{1} \times \mathbb{C P}_{1}$ is a smooth, totally real submanifold, and since $\tilde{f}\left(\partial D^{2}\right) \subset \tilde{N}$, it follows [1] that the non-constant holomorphic map $\tilde{f}$ cannot be constant to infinite order at any boundary point. Moreover, since $\tilde{N}$ is the graph of a diffeomorphism $\varphi: \mathbb{C P}_{1} \rightarrow \mathbb{C P}_{1}$, neither of the factor maps $\varpi_{j} \circ \tilde{f}, j=1,2$, can be constant to infinite order at a boundary point, either. Thus, setting $\amalg=\varpi_{1} \circ \tilde{f}$, letting $\zeta_{0} \in \partial D^{2}$ be any boundary point of the disk, and equipping $\mathbb{C P}_{1}$ with a local complex coordinate $z$ centered at $ц\left(\zeta_{0}\right)$, there must be an integer $k \geq 1$ such that

$$
ц(\zeta)=a\left(\zeta-\zeta_{0}\right)^{k}+O\left(\left|\zeta-\zeta_{0}\right|^{k+1}\right)
$$


for some $a \neq 0$, since the $C^{\infty}$ function ц satisfies the Cauchy-Riemann equations up to the boundary. If $k \geq 2$, it therefore follows that, in the fixed coordinate system, ц $(\stackrel{\circ}{D})$ must contain a punctured half-disk

$$
0<|z| \leq \epsilon, \quad \Re e\left(\mathrm{e}^{-\mathrm{i} \theta} z\right) \geq 0
$$

because the boundary of a wedge-shaped subregion

$$
0<\left|\zeta-\zeta_{0}\right|<\delta, \quad\left|\arg \left(\zeta-\zeta_{0}\right)-\theta\right|<\frac{2 \pi}{3 k}
$$

of $\stackrel{\circ}{D}^{2}$ is mapped by the smooth homeomorphism ц to a piece-wise $C^{1}$ Jordan curve with internal break-angle $4 \pi / 3>\pi$ at $ц\left(\zeta_{0}\right)$. However, the same argument can also be applied to the holomorphic map $\varpi_{2} \circ \tilde{f}$; thus, if $k \geq 2$, even after composing with the orientation-reversing diffeomorphism $\varphi^{-1}$, the boundary of an analogous wedge-shaped region would still be sent to a piece-wise $C^{1}$ Jordan curve whose internal break angle would have absolute value $>\pi$ at the origin, and the image of $\stackrel{\circ}{D}^{2}$ under the smooth map $\amalg=\varphi^{-1} \circ \varpi_{2} \circ \tilde{f}$ would therefore also contain a punctured half-disk

$$
0<|z| \leq \varepsilon, \quad \Re e\left(\mathrm{e}^{-\mathrm{i} \vartheta} z\right) \geq 0
$$

Since any two such punctured half-disks must meet, $k \geq 2$ thus implies that

$$
\text { ц }\left(\stackrel{\circ}{D}^{2}\right) \cap \amalg\left(\stackrel{\circ}{D}^{2}\right) \neq \varnothing,
$$

contradicting the previously established fact that $F$ is injective. Hence $k=1$, and, since $z_{0} \in \partial D^{2}$ is arbitrary, ц and $ш$ are both smooth embeddings $D^{2} \hookrightarrow \mathbb{C P}_{1}$. The parameterized holomorphic disk $\tilde{f}$ in $\left(\mathbb{C P}_{1} \times \mathbb{C P}_{1}, \tilde{N}\right)$ is therefore smoothly embedded, and the parameterized holomorphic disk $f$ in $\left(\mathbb{C P}_{2}, N\right)$ is therefore smoothly embedded, too.

If $L \rightarrow D^{2}$ is a complex line bundle over the disk, and if $\ell \rightarrow S^{1}$ is a real line sub-bundle of $\left.L\right|_{\partial D^{2}}$, recall [15] that the Maslov index ind $(L, \ell)$ is obtained by trivializing $L$, viewing $\ell$ as a map $\partial D^{2} \rightarrow \mathbb{R P}^{1}$ and declaring $\operatorname{ind}(L, \ell)$ to be the winding number of this map; the resulting integer is independent of the trivialization of $L$, and amounts [11] to the first Chern class of the double of $(L, \ell)$. More generally, if $V \rightarrow D^{2}$ is a rank- $r$ complex vector bundle, and if $V \rightarrow S^{1}$ is a rank- $r$ real sub-bundle of $\left.V\right|_{\partial D^{2}}$, the Maslov index ind $(V, V)$ is defined to be the Maslov index of the associated line-bundle pair $\left(\Lambda^{r} V, \Lambda^{r} V\right)$. 
If $Z$ is a complex manifold and $W \subset Z$ is a totally real submanifold, the total Maslov index of a parameterized holomorphic disk $f$ in $(Z, W)$ is defined to be $\operatorname{ind}\left(f^{*} T Z,\left(\left.f\right|_{\partial D^{2}}\right)^{*} T W\right)$. If the disk happens to be embedded, with image $\Delta=f\left(D^{2}\right)$, we also define the normal Maslov index of $\Delta$ to be ind $(N, n)$, where $N=T Z / T \Delta$ is the normal bundle of the disk, and $n=T W / T(\partial \Delta)$ is the relative normal bundle of its boundary. Since the Maslov index is additive, in the sense that

$$
\operatorname{ind}\left(V_{1} \oplus V_{2}, v_{1} \oplus V_{2}\right)=\operatorname{ind}\left(V_{1}, v_{1}\right)+\operatorname{ind}\left(V_{2}, V_{2}\right),
$$

and because ind $\left(T D^{2}, T\left(\partial D^{2}\right)\right)=2$, the total Maslov index of an embedded holomorphic disk equals its normal Maslov index plus two.

Proposition 2. Let $N \subset \mathbb{C P}_{2}$ be a docile surface, and let $f$ be a parameterized holomorphic disk in $\left(\mathbb{C P}_{2}, N\right)$ whose relative homology class represents the generator of $\mathrm{H}_{2}\left(\mathbb{C P}_{2}, N\right)$. Then $f$ has total Maslov index 3 , and its image $f\left(D^{2}\right)$ has normal Maslov index 1.

Proof. Given such a disk $f$, let $\tilde{f}: D^{2} \hookrightarrow \mathbb{C P}_{1} \times \mathbb{C P}_{1}$ be the branched lifting constructed in the proof of Proposition 1. Since the embedded holomorphic disk $\tilde{f}\left(D^{2}\right)$ is the graph of a diffeomorphism between domains in $\mathbb{C P}_{1}$ with smooth boundary, it is transverse to the first factor of $T \mathbb{C P}_{1} \times T \mathbb{C P}_{1}$, and its normal bundle can therefore be identified with the restriction of $T \mathbb{C P}_{1}$ to its first-factor projection $ц\left(D^{2}\right) \subset \mathbb{C P}_{1}$; this simultaneously identifies the relative normal bundle of the boundary with $\left(J \circ ц_{*}\right)\left[T\left(\partial D^{2}\right)\right]$. Taking an affine chart that contains ${ }^{2}\left(D^{2}\right)$, setting $\gamma=\left.ц\right|_{\partial D^{2}}$ and systematically exploiting the coordinate trivialization of $T \mathbb{C}$, the normal Maslov index of $\tilde{f}\left(D^{2}\right)$ therefore equals the winding number of

$$
\left(i \gamma^{\prime}\right)^{2}: S^{1} \rightarrow \mathbb{C}-\{0\}
$$

Since $\gamma$ is isotopic to the standard circle $\mathrm{e}^{\mathrm{i} \theta} \mapsto \mathrm{e}^{\mathrm{i} \theta}$ in $\mathbb{C},\left(i \gamma^{\prime}\right)^{2}$ is homotopic to $\mathrm{e}^{\mathrm{i} \theta} \mapsto \mathrm{e}^{2 \mathrm{i} \theta}$, and the normal Maslov index of $\tilde{f}\left(D^{2}\right)$ consequently equals 2 . On the other hand, $\tilde{f}\left(D^{2}\right)$ is a two-to-one branched over of $f\left(D^{2}\right)$, so the corresponding winding number for $f$ is only half as large, and the normal Maslov index of $f\left(D^{2}\right)$ therefore equals 1 . The total Maslov index of $f$ is therefore 3 , as claimed.

Theorem 1. Let $N \subset \mathbb{C P}_{2}$ be any docile surface, and let $p \in \mathcal{Q}$ be any point of the reference conic (1). Then there is a holomorphic disk in $\left(\mathbb{C P}_{2}, N\right)$ which passes through $p$ and represents the generator of $\mathrm{H}_{2}\left(\mathbb{C P}_{2}, N\right) \cong \mathbb{Z}$. Moreover, this disk is unique, modulo reparameterizations. 
Proof. By Lemma 1, there exists a smooth family of docile surfaces $N_{t}$, $t \in[0,1]$, such that $N_{1}=N$, and such that $N_{0}$ is the standard linear $\mathbb{R P}^{2} \subset$ $\mathrm{CP}_{2}$.

For the docile surface $N_{0}=\mathbb{R P}^{2}$, we can construct such a disk by using the complex conjugation map $\mathfrak{c}$ defined by (3). Indeed, since $\mathfrak{c}$ acts freely on $\mathcal{Q}$, the points $p$ and $\mathfrak{c}(p)$ are distinct, and hence joined by a unique projective line $\mathbb{C P}_{1} \subset \mathbb{C P}_{2}$. Since $p$ and $\mathfrak{c}(p)$ are interchanged by $\mathfrak{c}$, this projective line must be invariant under complex conjugation $\mathfrak{c}$, and it is therefore the complexification of a unique real projective line $\mathbb{R P}^{1} \subset \mathbb{R P}^{2}$. This $\mathbb{R P}_{1}$ divides the complex projective line $\mathbb{C P}_{1}$ into two disks, exactly one of which contains the given point $p \in \mathcal{Q}$; and since this disk meets $\mathcal{Q}$ transversely in a single point, its relative homology class generates $H_{2}\left(\mathbb{C P}_{2}, \mathbb{R P}^{2}\right)$. Conversely, the only disk with these properties is this half-projective-line. Indeed, if $\Delta$ is a disk in $\left(\mathbb{C P}_{2}, \mathbb{R P}^{2}\right)$ which passes through $p \in \mathcal{Q}$ and represents the generator of $H_{2}\left(\mathbb{C P}_{2}, \mathbb{R P}^{2}\right)$, then $\Delta \cup \mathfrak{c}(\Delta)$ is a holomorphic curve by the reflection principle, and, by Bézout's theorem, has degree one because it intersects the conic $\mathcal{Q}$ in two points. Thus, any holomorphic disk representing the generator of $H_{2}\left(\mathbb{C P}_{2}, \mathbb{R P}^{2}\right)$ is one hemisphere of a $\mathfrak{c}$-invariant complex projective line, with boundary a real projective line $\mathbb{R P}^{2} \subset \mathbb{R} \mathbb{P}^{2}$; and there is exactly one such hemisphere containing $p$. We have thus proved both existence and uniqueness for the "model" docile surface $N_{0}=\mathbb{R P}^{2}$.

We will now apply the continuity method to obtain an appropriate disk for each $t \in[0,1]$. Let $E \subset[0,1]$ be the set of $t$ for which such a disk exists; our goal is to show that $E=[0,1]$. We already know that $E \neq \varnothing$, since $0 \in E$. Because $[0,1]$ is connected, it therefore suffices to show that $E$ is both open and closed.

To show that $E$ is open, we appeal to the perturbation theory of holomorphic disks. Indeed, suppose that such a disk $\Delta$ exists for a certain value $\tau$ of $t$. By Proposition 1, $\Delta$ is smoothly embedded, and by Proposition 2, its normal Maslov index is 1 . The double of the normal bundle of $\Delta$, in the sense used in [11, Theorem 3], is therefore the $\mathcal{O}(1)$ line bundle over $\mathbb{C P}_{1}=\Delta \cup \bar{\Delta}$, and, since $H^{1}\left(\mathbb{C P}_{1}, \mathcal{O}(1)\right)=0$, the disk $\Delta$ is Fredholm regular. The moduli space $M_{\tau}$ of nearby holomorphic disks in $\left(\mathbb{C P}_{2}, N_{\tau}\right)$ is therefore smooth, with tangent space

$$
T_{\Delta} M_{\tau}=H_{\mathbb{R}}^{0}\left(\mathbb{C P}_{1}, \mathcal{O}(1)\right),
$$

where the right-hand side denotes the real-linear subspace of $H^{0}\left(\mathbb{C P}_{1}, \mathcal{O}(1)\right)$ consisting of sections which are real along $\mathbb{R P}^{1} \subset \mathbb{C P}_{1}$. Now observe that evaluation at two distinct points $p, q \in \mathbb{C P}_{1}$ gives rise to an isomorphism 
$H^{0}\left(\mathbb{C P}_{1}, \mathcal{O}(1)\right) \rightarrow \mathbb{C}^{2}$, and that evaluation at a single point $p \in \mathbb{C} \mathbb{P}_{1}-\mathbb{R P}^{1}$ therefore gives us a real-linear isomorphism $H_{\mathbb{R}}^{0}\left(\mathbb{C P}_{1}, \mathcal{O}(1)\right) \rightarrow \mathbb{C}$, as may be seen by setting $q=\bar{p}$. Since $\Delta$ is transverse to $\mathcal{Q}$ at $p \in \Delta$, it follows that the map $\varkappa_{\tau}: M_{\tau} \rightarrow \mathcal{Q}$ obtained by sending a disk to its intersection with $\mathcal{Q}$ has maximal rank at $\Delta$, and so is a diffeomorphism between a neighborhood of $\Delta \in M_{\tau}$ and a neighborhood of $p \in \mathcal{Q}$. This shows that, at least locally, $\Delta$ is the only disk in the family passing through the chosen point $p \in \mathcal{Q}$. Moreover, the Fredholm regularity of $\Delta$ guarantees that, for all $t$ in a neighborhood of $\tau$, there is a corresponding two-parameter family $M_{t}$ of embedded holomorphic disks in $\left(\mathbb{C P}_{2}, N_{t}\right)$, and the corresponding intersection map $\varkappa_{t}: M_{t} \rightarrow \mathcal{Q}$ is a local diffeomorphism onto a neighborhood of $p$. Consequently, for values of $t$ in an interval about $\tau$, there is a unique smooth family $\Delta_{t}$ of such holomorphic disks passing through $p$ with $\Delta_{\tau}=\Delta$. In particular, $E \subset[0,1]$ is open.

To show that $E$ is closed, we will now use a Gromov compactness argument. Indeed, suppose that $t_{j}$ is a sequence of values of $t \in[0,1]$ for which there exist a corresponding sequence of holomorphic disks $\Delta_{t_{j}}$ in $\left(\mathbb{C P}_{2}, N_{t_{j}}\right)$, each of which intersects $\mathcal{Q}$ transversely in the single point $p$; moreover, suppose that the numbers $t_{j}$ converge to some $\tau \in[0,1]$. Let $\tilde{\Delta}_{t_{j}}=\Pi^{-1}\left(\Delta_{t_{j}}\right)$ be the corresponding ramified lifts to $\mathbb{C P}_{1} \times \mathbb{C P}_{1}$. Each of these disks then meets the diagonal $\mathcal{D}=\Pi^{-1}(\mathcal{Q})$ transversely in the unique point $\tilde{p}$ corresponding to $p$. Consequently, each one represents the generator of $H_{2}\left(\mathbb{C P}_{1} \times \mathbb{C P}_{1}, \mathcal{D}\right)$. For $t \in[0,1]$, we now endow $\mathbb{C P}_{1} \times \mathbb{C P}_{1}$ with the smooth family of $\varrho$-invariant Kähler forms $\omega_{t}$ constructed in Lemma 3 ; this family is chosen so that $\tilde{N}_{t}$ is Lagrangian with respect to $\omega_{t}$, and all these forms $\omega_{t}$ belong to the same cohomology class, with respect to which each factor $\mathbb{C P}_{1}$ 's has area $4 \pi$. By Moser stability and the Weinstein tubular neighborhood theorem for Lagrangian submanifolds, the triples $\left(\mathbb{C P}_{1} \times \mathbb{C P}_{1}, \tilde{N}_{t}, \omega_{t}\right)$ are thus all symplectomorphic to the standard model $\left(\mathbb{C P}_{1} \times \mathbb{C P}_{1}, \tilde{N}_{0}, \omega_{0}\right)$, allowing us to think of the $\tilde{\Delta}_{t_{j}}$ as being a sequence of disks in $\left(\mathbb{C P}_{1} \times \mathbb{C P}_{1}, \tilde{N}_{0}, \omega_{0}\right)$ which are pseudo-holomorphic with respect to a sequence of $\omega_{0}$-compatible almost-complex structures. By Gromov compactness $[5,20]$, this sequence therefore has a subsequence which converges to a (possibly singular) pseudo-holomorphic curve $X$ in the same relative homology class, where $X$ is a union of holomorphic $\mathbb{C P}_{1}$ 's and at most one holomorphic disk in $\left(\mathbb{C P}_{1} \times \mathbb{C P}_{1}, \tilde{N}_{\tau}\right)$. Moreover, since $X$ is the Gromov limit of a sequence of $\varrho$-invariant curves through $\tilde{p}$, it must also be $\varrho$-invariant and pass through $\tilde{p}$. However, by construction,

$$
\omega_{\tau}=\varpi_{1}^{*} \check{\omega}_{\tau}+\varpi_{2}^{*} \check{\omega}_{\tau}
$$


for some area form $\check{\omega}_{\tau}$ on $\mathbb{C P}_{1}$. Since $X$ is invariant under the factorswitching involution $\varrho$ and represents the generator in $H_{2}\left(\mathbb{C P}_{1} \times \mathbb{C P}_{1}, \tilde{N}_{\tau}\right)$,

$$
2 \int_{X} \varpi_{1}^{*} \check{\omega}_{\tau}=\int_{X} \varpi_{1}^{*} \check{\omega}_{\tau}+\int_{X} \varpi_{2}^{*} \check{\omega}_{\tau}=\int_{X} \omega_{\tau}=4 \pi .
$$

The area, with multiplicities, of the projection of $X$ to either factor will thus be $2 \pi$, or in other words, half the area of the entire sphere. In particular, neither factor projection $X \rightarrow \mathbb{C P}_{1}$ can be onto. Consequently, $X$ cannot have any compact irreducible components, and is therefore a disk $\tilde{\Delta}_{\tau}$. Since $\tilde{\Delta}_{\tau}$ is $\varrho$-invariant, it must, moreover, be the ramified lift of a disk $\Delta_{\tau}$ in $\left(\mathbb{C P}_{2}, N_{\tau}\right)$. Because $\tilde{\Delta}_{\tau}$ meets the diagonal $\mathcal{D}$ transversely in the single point $\tilde{p}$, the disk $\Delta_{\tau}$ consequently meets $\mathcal{Q}$ transversely in the single point $p$. In particular, $\Delta_{\tau}$ passes through $p$ and represents the generator of $H_{2}\left(\mathbb{C P}_{2}, N_{\tau}\right)$. Thus $\tau \in E$, and $E$ is therefore closed.

Since $E \subset[0,1]$ has now been shown to be non-empty, open, and closed, the connectedness of the interval implies that $E=[0,1]$. In particular, $1 \in E$, so there exists a holomorphic disk in $\left(\mathbb{C P}_{2}, N\right)$ which passes through $p$ and represents the generator of $H_{2}\left(\mathbb{C P}_{2}, N\right)$, as claimed. It only remains for us to show that this disk is in fact unique.

To prove uniqueness, we apply the continuity method in reverse. Suppose that $\Delta^{\prime}$ is any holomorphic disk in $\left(\mathbb{C P}_{2}, N\right)$ which passes through $p$ and represents the generator of $H_{2}\left(\mathbb{C P}_{2}, N\right)$. By the same disk-perturbation argument as before, there is a unique smooth family $\Delta_{t}^{\prime}, t \in(\tau, 1]$, of holomorphic disks in $\left(\mathbb{C P}_{2}, N_{t}\right)$, meeting $\mathcal{Q}$ transversely in the single point $p$, such that $\Delta_{1}^{\prime}=\Delta^{\prime}$ and such that $\tau \in[0,1)$ is minimal. The above compactness argument then allows one to construct a limit disk $\Delta_{\tau}$, and perturbations of this disk allow one to extend the family across $t=\tau$. Since $\tau$ was taken to be as small as possible, this is a contradiction unless $\tau=0$. We thus actually obtain such a family of disks $\Delta_{t}^{\prime}$ for $t \in[0,1]$. However, $\Delta_{0}^{\prime}$ is then a disk in $\left(\mathbb{C P}_{2}, \mathbb{R P}^{2}\right)$ which meets $\mathcal{Q}$ transversely in the single point $p$, and we have already observed that this implies that $\Delta_{0}^{\prime}$ is one hemisphere of the projective line joining $p$ to $\mathfrak{c}(p)$. Thus, if there were two disks $\Delta$ and $\Delta^{\prime}$ in $\left(\mathbb{C P}_{2}, N\right)$, each meeting $\mathcal{Q}$ transversely in the single point $p$, both could be evolved backwards in $t$ to obtain the same disk. However, the process of evolving forward in $t$ starting with an initial disk at $t=0$ necessarily yields a unique final disk when $t=1$, so it follows that $\Delta^{\prime}=\Delta$. We have thus succeeded in establishing uniqueness, and our proof is therefore complete.

For clarity's sake, it is worth emphasizing that the above perturbation argument is carried out on the level of unparameterized embedded disks. 
For each unparameterized embedded disk, there is of course an $S L(2, \mathbb{R})$ 's worth of different parameterizations. The interested reader is invited to double-check our perturbation argument using the more popular machinery of parameterized disks and the total Maslov index [4, 15]. From this perspective, the moduli space of parameterized disks in $\left(\mathbb{C P}_{2}, N_{t}\right)$ near a given one will be five dimensional, and the moduli space of parameterized disks passing through $p$ will be three-dimensional. One can furthermore specialize the parameterization by requiring that $0 \in D^{2}$ be sent to $p$, and the resulting parameterized disk $f: D^{2} \rightarrow \mathbb{C P}_{2}$ will then be unique modulo rotations $f(\zeta) \rightsquigarrow f\left(e^{i \phi} \zeta\right)$.

Theorem 2. Let $N \subset \mathbb{C P}_{2}$ be any docile surface, and let $M$ denote the moduli space of all holomorphic disks in $\left(\mathbb{C P}_{2}, N\right)$ which represent the generator of $H_{2}\left(\mathbb{C P}_{2}, N\right) \cong \mathbb{Z}$. Then $M$ is diffeomorphic to $S^{2}$. The interiors of these disks foliate $\mathbb{C P}_{2}-N$, and the intersection pattern of their boundaries defines a unique Zoll projective structure $[\nabla]$ on $M$. Moreover, the reference conic $\mathcal{Q}$ induces a specific conformal structure $[g]$ on $M$, and there is a unique $\nabla \in[\nabla]$ which is a Weyl connection for the conformal class $[g]$.

Proof. Theorem 1 asserts that, through each $p \in \mathcal{Q}$, there is a unique holomorphic disk representing the generator of $H_{2}\left(\mathbb{C P}_{2}, N\right)$; moreover, the proof of this theorem shows the map $\varkappa: M \rightarrow \mathcal{Q}$, obtained by sending a disk to its intersection with the conic, to be a local diffeomorphism. Hence $\varkappa$ is actually a diffeomorphism, and $M \approx \mathcal{Q} \approx S^{2}$.

For each $x \in M$, let $\Delta_{x} \subset \mathbb{C P}_{2}$ be the embedded holomorphic disk it represents, and set

$$
\mathcal{F}=\left\{(x, y) \in M \times \mathbb{C P}_{2} \mid y \in \Delta_{x}\right\}
$$

Then the projection $(x, y) \mapsto x$ makes $\mathcal{F}$ into a smooth family of disks $p: \mathcal{F} \rightarrow M$. Let $\mathcal{B}$ be the Melrose blow-up of $\mathbb{C P}_{2}$ along $N$, which is the manifold-with-boundary obtained from $\mathbb{C P}_{2}$ by replacing each point of $N$ with the unit circle bundle in the normal bundle of $N$; let $b: \mathcal{B} \rightarrow \mathbb{C P}_{2}$ be the canonical projection, which we shall call the Melrose blow-down. Since each of the disks $\Delta_{x}$ is smoothly embedded, with $\partial \Delta_{x} \subset N$, the tautological smooth projection $q: \mathcal{F} \rightarrow \mathbb{C P}_{2}$ given by $(x, y) \mapsto y$ can be lifted to a smooth map $\hat{q}: \mathcal{F} \rightarrow \mathcal{B}$, with $q=b \circ \hat{q}$, by sending each boundary point to the radial derivative of $q$ relative to the corresponding disk. However, since each disk $\Delta_{x}$ has normal Maslov index 1, the normal bundle of $\Delta_{x}$ can be trivialized in such a manner that elements of the tangent space $T_{x} M$ are 
represented by some holomorphic function $\varsigma: D^{2} \rightarrow \mathbb{C}$ of the form

$$
\varsigma(\zeta)=a+\bar{a} \zeta
$$

for $a \in \mathbb{C}$ arbitrary. For $a \neq 0$, such a variation has a zero only at the boundary of the disk, and at this zero, its radial derivative is non-zero. Thus the derivative of $\hat{q}$ has maximal rank everywhere. Since the manifolds-withboundary in question are compact and connected, it follows that $\hat{q}$ is a covering map. However, $\mathcal{B}$ is diffeomorphic to the complement of a tubular neighborhood of $N$, and $\mathcal{Q} \hookrightarrow \mathcal{B}$ is therefore a homotopy equivalence by the proof of Lemma 2 . Thus $\mathcal{B}$ is simply connected, and $\hat{q}$ is therefore a diffeomorphism. In particular, it induces a diffeomorphism between the interiors of $\mathcal{F}$ and $\mathcal{B}$, so the interiors of the disks $\Delta_{x}$ foliate $\mathbb{C P}_{2}-N$, as claimed.

For any $y \in \mathcal{B}$ and $z=b(y)$, consider the pull-back map $b^{*}: \Lambda_{z}^{1,0} \rightarrow \Lambda_{y}^{1} \otimes$ $\mathbb{C}$. If $y$ is an interior point of $\mathcal{B}$, this is obviously injective, because $b$ is a local diffeomorphism near $y$. However, it remains injective even if $y$ is a boundary point. To see this, notice that, when $y \in \partial \mathcal{B}$, the kernel of the pull-back map $\Lambda_{z}^{1} \otimes \mathbb{C} \rightarrow \Lambda_{y}^{1} \otimes \mathbb{C}$ is one dimensional, and spanned by a real co-vector. Since $\Lambda_{z}^{1,0} \subset \Lambda_{z}^{1} \otimes \mathbb{C}$ contains no non-zero real vectors, it follows that $\Lambda_{z}^{1,0} \rightarrow \Lambda_{y}^{1} \otimes \mathbb{C}$ is injective, as claimed. Because $q=b \circ \hat{q}$, the annihilator of $b^{*}\left(\Lambda^{1,0}\right)$ therefore corresponds, via the diffeomorphism $\hat{q}$, to a rank-2 subbundle $Д \subset T_{\mathbb{C}} \mathcal{F}$, explicitly given by the kernel of $q_{*}^{1,0}: T_{\mathbb{C}} \mathcal{F} \rightarrow T^{1,0} \mathbb{C P}_{2}$. This sub-bundle is involutive on the interior of $\mathcal{F}$, and so, by continuity, is involutive even along $\partial \mathcal{F}$. However, since the derivative of $b$ has rank 3 at every boundary point, the same is true of $q$, and $Д=\operatorname{ker} q_{*}^{1,0}$ therefore contains a real direction at every point of $\partial \mathcal{F}$. It also contains the $(0,1)$ tangent space of the fiber disks of $p: \mathcal{F} \rightarrow M$, so we can apply [12, Lemma 4.6] to the double $\mathcal{F} \cup \overline{\mathcal{F}}$, exactly as in the proof of [12, Theorem 4.7]. Thus, there is a unique projective connection $[\nabla]$ on $\mathcal{M}$ for which the closed curves $\mathfrak{C}_{z}$, defined for $z \in N$ by

$$
\mathfrak{C}_{z}=p\left(q^{-1}[\{z\}]\right)=\left\{x \in \mathcal{M} \mid z \in \partial \Delta_{x}\right\}
$$

are the geodesics of $[\nabla]$. Moreover, since each disk-boundary $\partial \Delta_{x}$ is an embedded $S^{1} \subset N$, no fiber of $\left.p\right|_{\partial \mathcal{F}}$ can meet a fiber of $\left.q\right|_{\partial \mathcal{F}}$ in more than one point. Hence the $\mathfrak{C}_{z} \subset \mathfrak{M}$ are all simple closed curves, and $[\nabla]$ is therefore a Zoll projective structure on $M \approx S^{2}$. Finally, there is $[12$, footnote $4, \mathrm{p}$. $514]$ a unique choice of $\nabla \in[\nabla]$ which is a Weyl connection for the conformal structure on $M$ induced by its identification with $\mathcal{Q}$. 
Theorem 3. Let $N \subset \mathbb{C P}_{2}$ be a docile surface, and let $([g], \nabla)$ be the Weyl structure on $M \approx S^{2}$ whose existence is guaranteed by Theorem 2. Then $\nabla$ is the Levi-Civita connection of a Zoll metric $g \in[g]$ iff $N \subset \mathbb{C P}_{2}-\mathcal{Q}$ is Lagrangian with respect to the sign-ambiguous symplectic form $\Omega=\Im m \Upsilon$, where $\Upsilon$ is defined by Equation (2). When this happens, there is a unique such $g$ whose closed geodesics all have length $2 \pi$. This normalization is moreover equivalent to requiring that $\left(S^{2}, g\right)$ have total area $4 \pi$.

Proof. By Theorem 4.8 of [12], the Lagrangian condition is equivalent to the requirement that $\nabla$ be the Levi-Civita connection of a Zoll metric $g$. When this happens, $g$ is then of course determined up to an multiplicative constant, since $\nabla$ and $[g]$ are already known. We can moreover fix this scaling constant by specifying the length of some (and hence every) closed geodesic. However, it is also known [12, Theorem 2.15] that the geodesic flow of $g$ is differentiably conjugate to that of the standard metric $g_{0}$ on $S^{2}$. Hence Weinstein's theorem [18] predicts that the total area of $\left(S^{2}, g\right)$ will coincide with that of $\left(S^{2}, g_{0}\right)$ iff the closed geodesics of $g$ and $g_{0}$ have identical lengths.

Theorem 2 considerably clarifies our understanding of the return journey from totally real surfaces $N \subset \mathbb{C P}_{2}$ to Zoll projective structure $[\nabla]$ on $S^{2}$. As long as $N$ is docile with respect to some non-singular conic $\mathcal{Q}$, then there is a unique Zoll projective structure $[\nabla]$ on $S^{2}$ to which $N$ corresponds via the construction of [12]. However, because docility is an open condition, a surface $N$ which is docile with respect to a particular conic $\mathcal{Q}$ will also be docile with respect to a five-complex-parameter family of nearby conics $\mathcal{Q}^{\prime}$. Thus, every Zoll projective structure $[\nabla]$ arising from Theorem 2 can actually be represented by a 10-real-parameter family of Weyl connections $\nabla$, each compatible with a different conformal structure. However, moving $\mathcal{Q}^{\prime}$ far enough will always result in conics with respect to which a given $N$ will fail to be docile. For this reason, docility is best understood as a condition on Weyl connections rather than on projective structures.

One motivation for the present article stems from the fact that a Weyl structure $\left(S^{2},[g], \nabla\right)$ with all geodesics geometrically closed may be treated as time-symmetric Cauchy data for a Lorentzian Einstein-Weyl structure on $S^{2} \times \mathbb{R}$. From the twistor perspective, the latter arises as the moduli space of holomorphic disks in $\mathbb{C P}_{1} \times \mathbb{C P}_{1}$ with boundaries in $\tilde{N}=\Pi^{-1}(N)$. When $N$ is docile with respect to the branch locus $\mathcal{Q}$ of $\Pi$, our results from [13] guarantee long-time existence of the solution, and allow one to directly interpret the orientation-reversing involution $\varphi: \mathbb{C P}_{1} \rightarrow \mathbb{C P}_{1}$ in terms of the 
scattering of light rays in the resulting space-time. In this context, it would be fascinating to obtain a better understanding of the scattering map $\varphi$ directly in terms of the geometry of the initial-value surface. An attack on this initial-value problem by more direct methods might therefore offer new insights into the geometry of Zoll manifolds.

Of course, the most classical aspects of our present subject pertain not to Zoll projective structures, but rather to Zoll metrics. Since the conformal structure is intrinsically part of the geometry in this setting, it is completely natural in this context to fix a conic $\mathcal{Q} \subset \mathbb{C P}_{2}$ once and for all. Our previous work in [12] showed that every Zoll metric on $S^{2}$ gives rise to a Lagrangian surface $N \subset \mathbb{C P}_{2}-\mathcal{Q}$, and one might perhaps hope that this totally real submanifold would always turn out to be docile with respect to $\mathcal{Q}$. However, as we show in Appendix A below, this is not even true in the axisymmetric case. Thus, while we hope that the present paper offers some interesting advances in the theory of Zoll metrics, further new ideas and results will be needed in order, for example, to determine whether the space of Zoll metrics on $S^{2}$ is connected.

\section{Appendix A. The axisymmetric case}

The axisymmetric Zoll metrics can be given as

$$
d s^{2}=(1+F)^{2} d \theta^{2}+\sin ^{2} \theta d \phi^{2},
$$

where $F:=F(\theta)$ is odd about $\theta=\pi / 2, F(\pi-\theta)=-F(\theta)$ and the axisymmetry is generated by $\partial / \partial \phi$. For regularity at $\theta=0, \pi$, we assume that $F$ vanishes in some small neighborhood of these values. ${ }^{1}$

In this appendix, we will obtain an integral formula (A.11) for the principal piece of data in this metric, $F$, in terms of a function $a$ that controls the deformation of the real slice of $\mathbb{C P}^{2}$ appropriate to such an axisymmetric Zoll metric. In particular we will express the docility condition as a straightforward monotonicity condition (A.4) on $a$, and show that this guarantees that $1+F$ does not vanish. We will see however that docility is not a necessary condition; there are non-docile choices for $a$ that lead to a regular metric. Regularity requires that $|F|<1$ for $\theta \in[0, \pi]$ but we will see that docility of the real slice implies the stronger condition $|F|<|\sin \theta|$. If this latter inequality is violated for some value of $\theta$, the real slice must fail to be docile but this latter inequality can be violated with $|F|<1$.

\footnotetext{
${ }^{1}$ This is much stronger than necessary, but greatly simplifies the discussion.
} 
The calculation is analogous to that in the appendix of [12] in the projective case but here we restrict attention to the case where the projective structure arises from a metric, and we push the analysis one step further to obtain the integral formula (A.11) for $F$. We will also use a correspondence appropriate to the double cover $\mathbb{C P}^{1} \times \mathbb{C P}^{1}$, although we do not explore the corresponding extension to the Einstein-Weyl three-space in any detail. We first construct the holomorphic disks associated with a deformation of the real slice appropriate to the axisymmetric case. We find the corresponding metric by obtaining the geodesic spray, and so we digress briefly to introduce this.

The geodesic spray is the vector field on the unit tangent bundle $U T S^{2}$ that at $(v, x) \in U T_{x} S^{2}$ is the horizontal lift of the vector $v$ at $x$. We parametrize the fiber of $U T S^{2}$ so that $\zeta$ corresponds to the vector

$$
v=\frac{1}{\left(1+\zeta^{2}\right)}\left(2 \zeta \mathbf{e}_{1}+\left(\zeta^{2}-1\right) \mathbf{e}_{2}\right),
$$

where $\mathbf{e}_{1}$ and $\mathbf{e}_{2}$ are the orthonormal frame

$$
\left(\mathbf{e}_{1}, \mathbf{e}_{2}\right)=\left(\frac{1}{F+1} \frac{\partial}{\partial \theta}, \frac{1}{\sin \theta} \frac{\partial}{\partial \phi}\right) .
$$

Then the geodesic spray for the metric above is proportional to

$$
\Xi=\frac{\partial}{\partial \theta}+\frac{\zeta^{2}-1}{2 \zeta} \frac{F+1}{\sin \theta} \frac{\partial}{\partial \phi}-\frac{\zeta^{4}-1}{4 \zeta} \cot \theta \frac{\partial}{\partial \zeta} .
$$

(We must divide by $(F+1) 2 \zeta /\left(1+\zeta^{2}\right)$ to give the actual geodesic spray that gives the pathlength parameterization, but the above form will be more helpful for our purposes.) To see that the above gives a multiple of the geodesic spray, using the dual co-frame $\left(\mathbf{f}^{1}, \mathbf{f}^{2}\right)=((F+1) d \theta, \sin \theta d \phi)$, we first calculate the connection one-form to be

$$
\omega=\frac{\cot \theta}{F+1} \mathbf{f}^{2} \text {. }
$$

Thus the horizontal lift of $\mathbf{e}_{1}$ is simply $\mathbf{e}_{1}$ since $\omega\left(\mathbf{e}_{1}\right)=0$ but the horizontal lift of $\mathbf{e}_{2}$ is $\mathbf{e}_{2}-\omega\left(\mathbf{e}_{2}\right)\left(\zeta^{2}+1\right) \frac{\partial}{\partial \zeta}$ with our choice of parameterization of $U T S^{2}$. Thus the geodesic spray is

$$
\frac{2 \zeta}{1+\zeta^{2}} \mathbf{e}_{1}+\frac{\zeta^{2}-1}{1+\zeta^{2}}\left(\mathbf{e}_{2}-\left(1+\zeta^{2}\right) \frac{\cot \theta}{2(F+1)} \frac{\partial}{\partial \lambda}\right)
$$

and this is proportional to the formula given above as required. 
We now discuss the geometry of the deformed real slice in this axisymmetric context. We will use affine coordinates on $\mathbb{C P}^{1} \times \mathbb{C P}^{1}$ and $\mathbb{C P}^{2}$ related to the homogeneous coordinates used in the main text by

$$
u=\frac{u_{2}}{u_{1}}, \quad v=\frac{v_{2}}{v_{1}}, \quad z=\frac{\left(z_{2}+\mathrm{i} z_{1}\right)}{z_{3}}, \quad \tilde{z}=\frac{\left(z_{2}-\mathrm{i} z_{1}\right)}{z_{3}},
$$

with map $\mathbb{C P}^{1} \times \mathbb{C P}^{1} \rightarrow \mathbb{C P}^{2}$ given by

$$
(u, v) \rightarrow(z, \tilde{z})=\left(-\frac{2 u v}{u+v}, \frac{2}{u+v}\right)
$$

with the diagonal $u-v=0$ mapping to $z \tilde{z}+1=0$.

We introduce the invariant holomorphic coordinate

$$
w:=\frac{u+v}{u-v}= \pm \frac{1}{\sqrt{1+z \tilde{z}}} .
$$

The real slice corresponding to the standard metric is then $u=-1 / \bar{v}$ which maps to $\tilde{z}=\bar{z}$. The circle action is induced by the imaginary part of the holomorphic vector fields

$$
z \frac{\partial}{\partial z}-\tilde{z} \frac{\partial}{\partial \tilde{z}} \quad \text { or } \quad u \frac{\partial}{\partial u}+v \frac{\partial}{\partial v} .
$$

In terms of coordinates $(u, w)$, the real slice becomes

$$
w \in[-1,1] \quad \text { and } \quad|u|^{2}=\frac{1+w}{1-w} .
$$

In terms of the $(u, v)$ coordinates or $(u, w)$ coordinates, the symplectic form is

$$
\omega=\Re e \frac{d u \wedge d v}{(u-v)^{2}}=\Re e \frac{d w \wedge d u}{u} .
$$

If a Lagrangian deformation of the real slice is to be $S^{1}$-invariant, it must also map to the real interval $[-1,1]$ in the $w$-plane (i.e., the image of the real slice in the w-plane cannot change). This follows from the fact that the $S^{1}$ action is generated by the Hamiltonian $H=\Im m w$, so that if the orbits of the $S^{1}$ action lie in the surface, $H$ must be constant on the surface and we can take this constant to be zero by a re-definition of $w$.

There is still freedom to deform the real slice up and down the fibers of the projection to the $w$-plane, and the most general such can be specified in 
$(u, w)$ coordinates by

$$
|u|^{2}=\exp (a(w)) \frac{1+w}{1-w},
$$

where $a(w)$ is a real function on $[-1,1]$ with compact support inside the interval to ensure regularity at the fixed points $u=0, \infty$. (Of course, $a=0$ is the prototypical case.)

In order to restrict to the Zoll manifold case, our real slice must be invariant under the $\mathbb{Z}_{2}$ action that interchanges $u$ and $v$. This changes the sign of $w$ and $|u|^{2} \rightarrow|v|^{-2}$. Thus we require that $a$ be an even function of $w$. Thus, for an axisymmetric Zoll metric, the deformed real slice is given by $w \in[-1,1]$ and $u$ constrained by (A.3) for $a(w)$ an even compactly supported function of $w \in(-1,1)$.

We can easily choose this deformation to violate the docility condition. The real slice will hit a generator $u=$ constant twice if $a(w)$ is chosen so that $|u|$ has the same value for two different values of $w \in[-1,1]$; the fact that the symmetry orbits lie in the surface will look after the phase of $u$. However, this is easy to arrange as $a(w)$ is essentially arbitrary, we just need to choose $a(w)$ for $w \in[-1,1]$ such that for some $w_{1}, w_{2} \in[-1,1], w_{1} \neq w_{2}$ and

$$
\mathrm{e}^{a\left(w_{1}\right)} \frac{1+w_{1}}{1-w_{1}}=\mathrm{e}^{a\left(w_{2}\right)} \frac{1+w_{2}}{1-w_{2}}
$$

although this cannot be done with $a(w)$ small.

The docility condition can be expressed as the condition that the function $\exp (a(w))(1+w) /(1-w)$ be strictly monotone. This is equivalent, upon taking logs and differentiating, to

$$
a^{\prime}+\frac{2}{1-w^{2}}>0
$$

and because $a^{\prime}$ is odd and the second term even in $w \rightarrow-w$ we obtain

$$
\left|a^{\prime}\right|<\frac{2}{1-w^{2}} .
$$

There is an obvious possibility for pathology in the solution when $1+$ $F=0$ and so we would like to see that this does not occur if (A.4) holds.

We at first set up the correspondence in somewhat greater generality than we need for the Zoll surface case, and describe the correspondence between points of de Sitter space and discs in $\mathbb{C P}^{1} \times \mathbb{C P}^{1}$. With coordinates 
$(t, \theta, \phi)$ the standard de Sitter metric can be written as

$$
d s^{2}=\frac{\mathrm{d} t^{2}}{1+t^{2}}-\left(1+t^{2}\right)\left(d \theta^{2}+\sin ^{2} \theta d \phi^{2}\right)
$$

It will be convenient to use a time coordinate $\tau$ that is adapted to the conformal compactification defined by $t=\cot \tau$ so that

$$
d s^{2}=\frac{1}{\sin ^{2} \tau}\left(d \tau^{2}-d \theta^{2}-\sin ^{2} \theta d \phi^{2}\right), \quad \tau, \theta \in[0, \pi]
$$

and we have that $t= \pm \infty$ corresponds to $\tau=0, \pi$.

The holomorphic disks corresponding to points of de Sitter space lie in the Riemann spheres defined implicitly by the equation

$$
\cos \tau(u-v)+\cos \theta(u+v)+\sin \theta\left(\mathrm{e}^{\mathrm{i} \phi} u v-\mathrm{e}^{-\mathrm{i} \phi}\right)=0
$$

We will find an explicit parametric version to be more useful in which:

$$
(u, v)=\mathrm{e}^{-\mathrm{i} \phi}\left(\frac{\sin \sigma_{+} \zeta+\mathrm{i} \sin \sigma_{-}}{\cos \sigma_{+} \zeta-\mathrm{i} \cos \sigma_{-}},-\frac{\cos \sigma_{+} \zeta+\mathrm{i} \cos \sigma_{-}}{\sin \sigma_{+} \zeta-\mathrm{i} \sin \sigma_{-}}\right),
$$

where $\zeta$ is taken to be in the upper-half plane and

$$
\sigma_{ \pm}=\frac{\tau \pm \theta}{2}
$$

The boundaries of these disks project to give a $1: 1$ cover of the complement of the interval between $-\cos 2 \sigma_{-}$and $-\cos 2 \sigma_{+}$in the $w$ Riemann sphere:

$$
w=-\frac{\cos 2 \sigma_{+} \zeta^{2}+\cos 2 \sigma_{-}}{\zeta^{2}+1} \quad \Leftrightarrow \quad \zeta=\sqrt{\frac{\cos 2 \sigma_{+}+w}{w+\cos 2 \sigma_{-}}}
$$

For simplicity, we henceforth restrict to the Zoll-metric slice for which $\tau=\pi / 2$, which entails $\sigma_{+}=\pi / 2-\sigma_{-}=\pi / 4+\theta / 2$, and we use the variable $\sigma:=\sigma_{-}$in place of $\sigma_{ \pm}$. The formulae for the holomorphic disks reduce to

$$
(u, v)=\mathrm{e}^{-\mathrm{i} \phi}\left(\frac{\cos \sigma \zeta+\mathrm{i} \sin \sigma}{\sin \sigma \zeta-\mathrm{i} \cos \sigma},-\frac{\sin \sigma \zeta+\mathrm{i} \cos \sigma}{\cos \sigma \zeta-\mathrm{i} \sin \sigma}\right),
$$


and the projections to the $w$-plane become

$$
w=\sin \theta \frac{\zeta^{2}-1}{\zeta^{2}+1} \quad \Leftrightarrow \quad \zeta=\sqrt{\frac{w-\sin \theta}{w+\sin \theta}},
$$

branching at $\pm \sin \theta$.

In the deformed case, we wish to construct holomorphic disks whose boundaries lie in the deformed submanifold determined by (A.3). This can be reduced to an abelian problem. The real function $a(w)$ is defined on $[-1,1]$. Expressed as a function of $\zeta$ via (A.7) it becomes a real function on the real axis. We can express it as the real part of a holomorphic function

$$
a\left(\sin \theta \frac{\zeta^{2}-1}{\zeta^{2}+1}\right)=g+\bar{g}
$$

for $g:=g(\zeta, \theta)$ holomorphic on the upper-half plane in $\zeta$. This can be obtained by the standard Laurent theorem argument from the Cauchy integral formula

$$
\begin{aligned}
g(\zeta, \theta)= & \frac{1}{2 \pi \mathrm{i}} \int_{-\infty}^{\infty} \frac{d \eta}{\eta-\zeta} a\left(\sin \theta \frac{\eta^{2}-1}{\eta^{2}+1}\right) \\
& -\frac{1}{2 \pi \mathrm{i}} \mathrm{P} . \mathrm{V} \cdot \int_{-\infty}^{\infty} \frac{d \eta}{\eta-1} a\left(\sin \theta \frac{\eta^{2}-1}{\eta^{2}+1}\right),
\end{aligned}
$$

where the second term is an additive constant that fixes the ambiguity in the choice of $g$ by requiring that $g(1, \theta)=0$.

The disks are then obtained as

$$
(u, v)=\mathrm{e}^{g-\mathrm{i} \phi}\left(\frac{\cos \sigma \zeta+\mathrm{i} \sin \sigma}{\sin \sigma \zeta-\mathrm{i} \cos \sigma},-\frac{\sin \sigma \zeta+\mathrm{i} \cos \sigma}{\cos \sigma \zeta-\mathrm{i} \sin \sigma}\right),
$$

since then, for $\zeta$ real,

$$
|u|^{2}=\mathrm{e}^{g+\bar{g}} \frac{1+w}{1-w}=\mathrm{e}^{a(w)} \frac{1+w}{1-w}
$$

as required for the deformed real slice (A.3).

In order to construct the corresponding metric on the $S^{2}$ space of such disks, we need to find how the geodesic spray $\Xi$ is deformed away from that given in (A.2). In our context, the boundaries of the holomorphic disks that we have constructed form an $S^{1}$ bundle over $S^{2}$ that is naturally identified 
with the unit tangent bundle $U T S^{2}$ of $S^{2}$. The geodesic spray is the vector field tangent to the projection from this space to the deformed real slice of $\mathbb{C P}^{1} \times \mathbb{C P}^{1}$. Since the projection to the interval $[-1,1]$ in the $w$ plane is undeformed, we can project the geodesic spray from $U T S^{2}$ to the quotient by $\frac{\partial}{\partial \phi}$ to obtain the vector field $\Xi^{\prime}$ and this will be the same as in the standard case, both by inspection of (A.2) and because it must satisfy $\Xi^{\prime} w=0$ on the real slice, but the maps of the holomorphic disks onto the $w$-plane are the same as in the standard case. Thus

$$
\Xi^{\prime}=\frac{\partial}{\partial \theta}-\cot \theta \frac{\zeta^{4}-1}{4 \zeta} \frac{\partial}{\partial \zeta}
$$

The full geodesic spray $\Xi$ is determined by the condition that $\Xi u=0$ so that

$$
\Xi=\Xi^{\prime}-\mathrm{i} \frac{\Xi^{\prime} u}{u} \frac{\partial}{\partial \phi} .
$$

Substituting in (A.9) we obtain

$$
-\mathrm{i} \frac{\Xi^{\prime} u}{u}=-\mathrm{i} \Xi^{\prime} g+\frac{\zeta^{2}-1}{2 \zeta \sin \theta} .
$$

The integral formula (A.8) for $g$ gives, after some manipulation ${ }^{2}$

$$
-\mathrm{i} \Xi^{\prime} g=-\frac{\zeta^{2}-1}{2 \zeta} \frac{\cos \theta}{\pi} \int_{-\infty}^{\infty} \frac{\eta^{2} a^{\prime}\left(\sin \theta \frac{\eta^{2}-1}{\eta^{2}+1}\right) d \eta}{\left(\eta^{2}+1\right)^{2}}
$$

Comparing (A.10) with (A.2) and making the trigonometric substitution $\eta=\tan \psi / 2$ we obtain the integral formula

$$
F=-\frac{\sin 2 \theta}{8 \pi} \int_{-\pi}^{\pi}(1-\cos \psi) a^{\prime}(\sin \theta \cos \psi) d \psi .
$$

Finally, we note that the docility condition (A.4) can be used to guarantee the regularity of the metric in this context by observing that for $\theta \in[0, \pi / 2]$

$$
1=\frac{\cos \theta}{2 \pi} \int_{-\pi}^{\pi}(1-\cos \psi) \frac{1}{1-\sin ^{2} \theta \cos ^{2} \psi} d \psi
$$

\footnotetext{
${ }^{2}$ One integrates by parts on the term that arises from the $\zeta$ derivative in $\Xi^{\prime}$ to differentiate the $a$ in this term with respect to $\eta$. The involutions $\eta \rightarrow-\eta, \eta \rightarrow-1 / \eta$ together with the oddness of $a^{\prime}$ are used to simplify the formula further.
} 
so that

$$
\begin{aligned}
\sin \theta \mp F= & \frac{1}{16 \pi} \int_{-\pi}^{\pi}(1-\cos \psi) \sin 2 \theta \\
& \times\left( \pm a^{\prime}(\sin \theta \cos \psi)+\frac{2}{1-\sin ^{2} \theta \cos ^{2} \psi}\right) d \psi
\end{aligned}
$$

Thus for $\theta \in[0, \pi / 2]$ the right and hence left-hand sides are manifestly positive if the docility condition (A.4) is satisfied. Since $F$ is odd under $\theta \rightarrow \pi / 2-\theta$ we obtain

$$
|F|<\sin \theta \quad \theta \in[0, \pi]
$$

This is clearly more than sufficient to prevent the vanishing of $1+F$ on $[0, \pi]$; any $F$ with $1>|F|$ on $[0, \pi]$, but with $|F|>|\sin \theta|$ at some $\theta \in[0, \pi]$ corresponds to a regular Zoll metric that arises from a non-docile real slice.

\section{Acknowledgments}

C.L. would like to thank Chris Bishop and Dennis Sullivan for their helpful explanations of relevant aspects of the theory of quasi-conformal mappings. We would also like to offer our hearty thanks to Frédéric Rochon and an anonymous referee for suggesting a number of important minor corrections.

C.L. was supported in part by NSF grant DMS-0905159. L.J.M. was supported in part by the EU under the FP6 Marie Curie Research Training Network ENIGMA (contract number MRTN-CT-2004-5652).

\section{References}

[1] S. Alinhac, M.S. Baouendi and L.P. Rothschild, Unique continuation and regularity at the boundary for holomorphic functions, Duke Math. J. 61 (1990), 635-653.

[2] A.L. Besse, Manifolds all of whose geodesics are closed, Springer-Verlag, Berlin, 1978.

[3] E.M. Chirka, Regularity of the boundaries of analytic sets, Mat. Sb. (N.S.) 117(159) (1982), 291-336, 431.

[4] F. Forstnerič, Analytic disks with boundaries in a maximal real submanifold of $\mathbf{C}^{2}$, Ann. Inst. Fourier (Grenoble) 37 (1987), 1-44. 
[5] U. Frauenfelder, Gromov convergence of pseudoholomorphic disks, J. Fixed Point Theory Appl. 3 (2008), 215-271.

[6] P. Funk, Über flächern mit lauter geschlossenen geodätischen Linien, Math. Ann. 74 (1913), 278-300.

[7] L.W. Green, Auf wiedersehensflächen, Ann. Math. (2) 78 (1963), 289299.

[8] D. Gromoll and K. Grove, On metrics on $S^{2}$ all of whose geodesics are closed, Invent. Math. 65 (1981/82), 175-177.

[9] K. Grove and W. Ziller, Cohomogeneity one manifolds with positive Ricci curvature, Invent. Math. 149 (2002), 619-646.

[10] V. Guillemin, The Radon transform on Zoll surfaces, Adv. Math. 22 (1976), 85-119.

[11] C. LeBrun, Twistors, holomorphic disks, and Riemann surfaces with boundary, in 'Perspectives in Riemannian geometry', eds. V. Apostolov, A. Dancer, N. Hitchin \& M. Wang, CRM Proc. Lecture Notes, 40, Amer. Math. Soc., Providence, RI, 2006, 209-221.

[12] C. LeBrun and L.J. Mason, Zoll manifolds and complex surfaces, J. Diff. Geom. 61 (2002), 453-535.

[13] C. LeBrun and L.J. Mason, The Einstein-Weyl equations, scattering maps, and holomorphic disks, Math. Res. Lett. 16 (2009), 291-301.

[14] O. Lehto and K.I. Virtanen, Quasiconformal mappings in the plane, Springer-Verlag, New York, 2nd ed., 1973.

[15] D. McDuff and D. Salamon, J-holomorphic curves and symplectic topology, Amer. Math. Soc., Providence, RI, 2004.

[16] S. Rickman, Quasiregular mappings, Springer-Verlag, Berlin, 1993.

[17] F. Rochon, On the uniqueness of certain families of holomorphic disks, 2007, e-print arXiv:0709.1118 [math.DG].

[18] A. Weinstein, On the volume of manifolds all of whose geodesics are closed, J. Diff. Geom. 9 (1974), 513-517.

[19] S.T. Yau, Calabi's conjecture and some new results in algebraic geometry, Proc. Natl. Acad. USA 74 (1977), 1789-1799.

[20] R. Ye, Gromov's compactness theorem for pseudo holomorphic curves, Trans. Amer. Math. Soc. 342 (1994), 671-694. 
[21] O. Zoll, Über Flächen mit Scharen geschlossener geodätischer Linien, Math. Ann. 57 (1903), 108-133.

Mathematics Department

SUNY, STONY BROOK

NY 11794

USA

E-mail address: claude@math.sunysb.edu

Mathematical Institute

24-29 ST. GILES

OXFORD OX1 3LB

$\mathrm{UK}$

E-mail address: lmason@maths.ox.ac.uk

Received February 15, 2010 